\title{
Update on degenerative spondylolisthesis: surgical treatment
}

\section{Atualização em eSPondilolistese degenerativa: tratamento CiRúrgico}

Bernardo WM, Botelho RV, Foni NO, Gotfryd AO, Herrero CFPS, Jefferson D, Meves R, Mudo ML, Simões RS,

ZYLBERSZTEJN S

http://dx.doi.org/10.1590/1806-9282.60.05.003

1. Is the use of bone substitutes such as BMP (bone morphogenetic protein) safe and effective in lumbosacral arthrodesis?

a. It is safe due to lack of complications.

b. Complications include osteolysis and heterotopic ossification.

c. The routine use is recommended in spondylolisthesis.

d. Clinical results with iliac grafts are superior.

2. Bone substitutes are equal or superior to autografts in this situation?

a. The removal of autologous bone graft from the iliac bone is radiologically superior.

b. Bone substitutes are contraindicated in this clinical situation.

c. The association of local bone graft (from the posterior vertebral elements) and beta-tricalcium phosphate is a therapeutic option.

d. The removal of autologous bone graft from the iliac bone is clinically superior.

3. What is the most appropriate diagnostic study in this clinical context?

a. In the presence of symptomatic lumbar stenosis, the most sensitive and specific radiologic examination is computed tomography (CT).

b. Plain radiographs in the standing position does not determine the diagnosis.

c. Magnetic Resonance Imaging (MRI) is more sensitive and specific in identifying the narrowing of the spinal canal.

d. The most appropriate diagnostic test in this situation is plain radiography.
4. Is it necessary to refer the patient to arthrodesis with use of rigid pedicle screws (non-dynamic)?

a. Instrumentation does not increase fusion rate.

b. Instrumented fusion produces less progression and improved walking ability.

c. A side effect of fusion is increased radicular pain.

d. There is subjective improvement independent of solid fusion.

5. How long should conservative treatment (non-surgical) be maintained?

a. In treatment failure after 1 year of therapy.

b. Medical and surgical treatments have similar results, differing in required time.

c. After 12 weeks of failure in conservative treatment.

d. Spondylolisthesis is an absolute indication for surgical treatment. 